\title{
Development of a first-generation miniature multiple reference optical coherence tomography imaging device
}

Paul M. McNamara

Roshan Dsouza

Colm O'Riordan

Seán Collins

Peter O'Brien

Carol Wilson

Josh Hogan

Martin J. Leahy 


\title{
Development of a first-generation miniature multiple reference optical coherence tomography imaging device
}

\author{
Paul M. McNamara, ${ }^{\mathrm{a}, \mathrm{b}, *}$ Roshan Dsouza, ${ }^{\mathrm{a}, \mathrm{b}}$ Colm O'Riordan, ${ }^{\mathrm{c}}$ Seán Collins, ${ }^{\mathrm{c}}$ Peter O'Brien, ${ }^{\mathrm{c}}$ Carol Wilson, ${ }^{\mathrm{b}}$ \\ Josh Hogan, ${ }^{b}$ and Martin J. Leahy ${ }^{a}$ \\ ${ }^{a}$ National University of Ireland, School of Physics, Tissue Optics and Microcirculation Imaging Group, National Biophotonics and Imaging Platform, \\ Galway H91 CF50, Ireland \\ ${ }^{\mathrm{b} C o m p a c t ~ I m a g i n g ~ I n c ., ~} 897$ Independence Avenue, Suite 5B, Mountain View, California 94043, United States \\ 'Irish Photonic Integration Centre (IPIC), Tyndall National Institute, Lee Maltings Complex, Dyke Parade, Cork T12 R5CP, Ireland
}

\begin{abstract}
Multiple reference optical coherence tomography (MR-OCT) is a technology ideally suited to low-cost, compact OCT imaging. This modality is an extension of time-domain OCT with the addition of a partial mirror in front of the reference mirror. This enables extended, simultaneous depth scanning with the relatively short scan range of a miniature voice coil motor on which the scanning mirror is mounted. This work details early stage development of the first iteration of a miniature MR-OCT device. This iteration utilizes a fiber-coupled input from an off-board superluminescent diode. The dimensions of the module are $40 \times 57 \mathrm{~mm}$. Off-the-shelf miniature optical components, voice coil motors, and photodetectors are used, with the complexity of design depending on the specific application. The photonic module can be configured as either polarized or nonpolarized and can include balanced detection. The results shown in this work are from the nonpolarized device. The system was characterized through measurement of the input spectrum, axial resolution, and signal-to-noise ratio. Typical B-scans of static and in vivo samples are shown, which illustrate the potential applications for such a technology. ( 2016 Society of Photo-Optical Instrumentation Engineers (SPIE) [DOI: 10.1117/1.JBO.21.12.126020]
\end{abstract}

Keywords: multiple reference optical coherence tomography; miniature optical systems; low cost; portable; low coherence; biometrics. Paper 160647PR received Sep. 19, 2016; accepted for publication Dec. 7, 2016; published online Dec. 28, 2016.

\section{Introduction}

Optical coherence tomography (OCT) is a recently developed imaging technology that has the ability to noninvasively visualize three-dimensional (3-D) structural information of a biological sample on the scale of microns. ${ }^{1}$ Generally, the method is based on a Michelson-type interferometric set up that utilizes a broadband light source to coherence-gate light, which is scattered from the sample. In time-domain OCT (TD-OCT), the reference mirror is longitudinaly translated in time to provide remote depth scanning of the sample. Spectral-domain OCT (SD-OCT) and swept-source OCT (SSOCT) systems utilize a spectrometer and frequency swept laser source, respectively, to acquire a depth-resolved interference signal without the necessity of scanning a reference mirror. Both SD-OCT and SS-OCT have been shown to have numerous advantages over TD-OCT in terms of speed and sensitivity. Commercially, OCT systems have been shown to have a diverse range of clinical and surgical applications in areas such as opthalmology, cardiology, oncology, and dermatology, among others ${ }^{2}$ however, to date, commercial systems have been large and expensive. Successful development of a miniature and affordable version of this technology opens the door to completely novel fields of application. Recently, other groups have reported the development of silicon-based integrated systems for SD-OCT ${ }^{3,4}$ and SS-OCT. ${ }^{5,6}$ However, swept source lasers and InGaAs cameras remain expensive, which is currently limiting

*Address all correspondence to: Paul M. McNamara, E-mail: paul.mcnamara@ nuigalway.ie the development of consumer-level SS-OCT and SD-OCT devices.

Multiple reference OCT (MR-OCT) is an extension of TDOCT through the addition of a partial mirror in front of the reference mirror. This recirculation of light between the partial mirror and reference mirror results in multiple orders of reflection, which can interfere with the light coming from the sample over an extended depth range. This combination allows simultaneous scanning of multiple segments, successively deeper in depth by scanning the reference mirror a relatively short distance $(\sim 30$ to $40 \mu \mathrm{m}$ ). The MR-OCT approach enables the use of low cost, off the shelf mechanical components such as voice coils that have been shown to be effective miniature, low-voltage actuators. ${ }^{7-9}$ Hence, this technology has the potential to produce a fully functional OCT system (excluding processing and display) with a bill of materials of approximately $\$ 10 .{ }^{10}$ Furthermore, we expect MR-OCT devices to be available before photonic integrated circuit-based devices reach their full potential.

There exists a multitude of potential applications for consumer-level OCT technology. One example is the use of OCT for the purposes of enhancing fingerprint technology. ${ }^{11-14}$ The presence of a subsurface fingerprint (sometimes called the primary fingerprint) at the epidermis-dermis boundary, which is an exact copy of the surface (secondary) fingerprint, means that detection of descrepencies between the two patterns can signify the presence of an artificial fingerprint. Liu et al. has also shown that visualizing the distribution of sweat (eccrine)

$1083-3668 / 2016 / \$ 25.00$ @ 2016 SPIE 


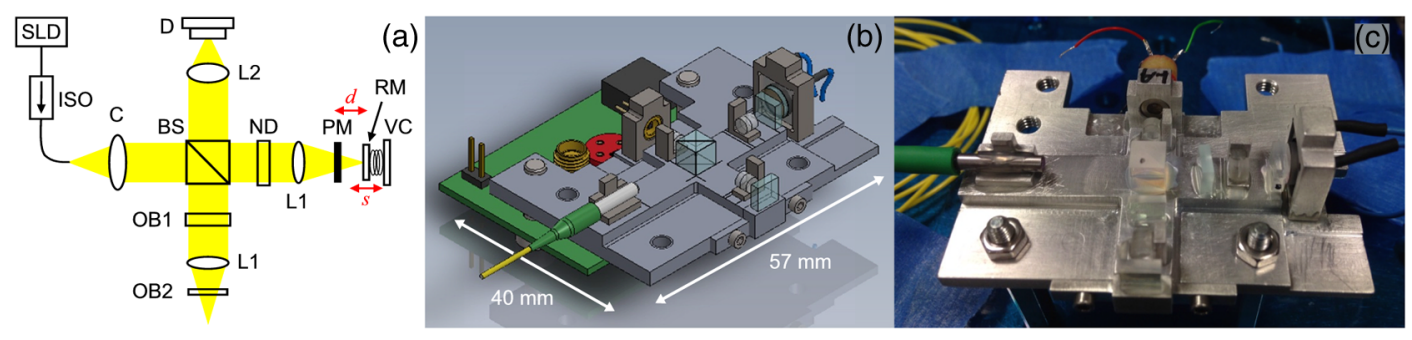

Fig. 1 (a) Schematic of the MR-OCT set up. ${ }^{20}$ SLD, superluminescent diode; ISO, optical isolator; C, collimator; BS, beam splitter (50:50); ND, neutral density filter $(\mathrm{OD}=0.5)$; $\mathrm{L} 1$, sample and reference focusing lenses $(E F L=6 \mathrm{~mm}$, spot size $=30 \mu \mathrm{m})$; PM, partial mirror $(80 \%$ reflectivity on the reference mirror side); RM, reference mirror; VC, voice coil (oscillation of $152 \mathrm{~Hz}$ ); OB1, optical blank 1 (compensation for ND filter); OB2, optical blank 2 (compensation for partial mirror); L2, detector lens (EFL = $6 \mathrm{~mm}$, spot size $=30 \mu \mathrm{m}$ ); D, photo detector (active area diameter $=500 \mu \mathrm{m}$ ). (b) SolidWorks drawing of the miniature MR-OCT device. Note that the components ND and OB1 are not included in this drawing. (c) Photograph of the miniature MR-OCT device.

glands enhances the accuracy of fingerprint detection. ${ }^{15}$ In addition to structural information, the ability of OCT to detect functional information such as blood flow, heart rate, and perspiration contributes to liveness detection and can potentially contribute to a very secure biometric system. ${ }^{16}$ Until now, the capabilities of OCT to provide fingerprint authentication information have been irrelevant because OCT systems have been too large and expensive to deploy to field applications.

Another field that would benefit hugely from a consumerlevel OCT device is opthalmology. Because of the availability of photodetectors across a wide range of wavelengths, MR-OCT can be designed for both $1300 \mathrm{~nm}$ for tissue imaging and $850 \mathrm{~nm}$ for ophthalmic applications. For example, the usefulness of OCT for monitoring the progress and treatment of age-related macular degeneration, a leading cause of blindness, has been well documented in the literature. ${ }^{17-19}$ An MR-OCT device could potentially take the form of a home-based monitoring system, in which the user could take daily scans that could automatically record thickness measurements of their retina and relay the information to their practitioner.

Other potential applications of this technology, previously demonstrated by the Tissue Optics and Microcirculation Imaging Group at NUI Galway, include dermatology, ${ }^{7}$ nondestructive testing, ${ }^{9}$ and dentistry. ${ }^{8}$ All of these works were performed via bench-top systems using bulk optics. This current work describes the first steps in the transition to a truly miniature OCT system. Two versions of the miniature module (unpolarized and polarized) have been thus far developed, the first of which is described in this work. This module is an unpolarized version with a very simple design of just eleven components. A schematic and image of the device can be seen in Fig. 1.

\section{Experimental Set Up and Methods}

\subsection{Optical Design}

Assembly of the module was done using the NanoGlue active alignment workstation by Nanosystec. ${ }^{21}$ The NanoGlue is composed of two independent stages, which use a customized gripper appendage to pick and place optical components onto the substrate. Each stage has six degrees of freedom $(x, y, z$, pitch, roll, and yaw) with a linear and rotary axis resolution of $20 \mathrm{~nm}$ and $0.0005 \mathrm{deg}$, respectively. A vertically mounted camera provides machine vision capability and visual feedback to the operator. A scanning slit beam profiler (Thorlabs, BP209$\mathrm{IR} / \mathrm{M})$ is mounted on a translation stage, which provides beam profiling capabilities as well as simple off-board optical power detection of NIR wavelengths. Each component was glued to the substrate using a low shrinkage UV cured adhesive (Dymax, 6-621-VT).

The light source used in this device is a Denselight (DLCS3184A) superluminescent diode (SLD). This source has a central wavelength, $\lambda_{0}$ of $1310 \mathrm{~nm}$, with a bandwidth, $\Delta \lambda$ of $40 \mathrm{~nm}$, giving an axial resolution $\Delta z \sim 19 \mu \mathrm{m}$. The light from the SLD is collimated using a fiber collimator, which is mounted directly on the module giving a beam diameter of $350 \mu \mathrm{m}$. A 5-mm beam splitter is used to split the input beam into the sample and reference paths. The sample and reference paths contain an achromat focusing lens (Ross Optical, L-MAC000) with a 3-mm diameter, resulting in a spot size of $30 \mu \mathrm{m}$ and a Rayleigh range of $570 \mu \mathrm{m}$. On the reference arm, a partial mirror having a reflectivity $80 \%$ is placed a distance of $100 \mu \mathrm{m}$ in front of the reference mirror. The reference mirror is mounted on a voice coil (Alps, ATMC1Z301A). The detection side of the design consists of a focusing lens and an InGaAs photodiode detector (ThorLabs, FDGA05) with a $500 \mu \mathrm{m}$ active area. The photo detector is connected to a custom designed transimpedance amplifier, which sits underneath the optical bench. All surfaces of the optical components (except for the reference mirror and partial mirror) are AR coated.

During assembly of this photonic module, it was observed that the overall performance is heavily dependent on the movement of the voice coil. The pointing stability of a particular voice coil affects the quality of the interference fringes observed at the detector. This sensitivity was alleviated somewhat through the use of focusing lenses on the sample and reference arms. Operation of the voice coil is based on a sinusoidal input voltage at $152 \mathrm{~Hz}$ with a scan range of $\sim 35 \mu \mathrm{m}$. Tuning the DC offset is of vital importance since this has an effect on the spacing between the partial mirror and reference mirror and hence the separation between consecutive orders of reflection from the reference mirror. ${ }^{22}$ It should be highlighted that the voice coil used in this work was being used outside of its design parameters. This particular voice coil was designed for focusing a lens in the camera of a mobile phone and does not normally operate in a bipolar mode. For this reason, tuning is required. In future iterations, a custom voice coil with improved pointing stability will be used.

The substrate on which all the optical components were placed is made of aluminum with dimensions of $40 \times 57 \mathrm{~mm}$. The design of the substrate included a raised central "rail" on 
which the main optical components were placed. Mechanical mounts for the optical components were custom designed and manufactured.

\subsection{Alignment Procedure}

Extensive planning went into the design of the alignment procedure. Since it was not possible to remove or alter the orientation of individual components after gluing, the sequence of adding components had to be planned within the constraint of allowing spatial clearance of the arms of the NanoGlue system at each step. In terms of the alignment of each of the individual optical components, a number of methods were used to optimize the position and tip/tilt of each component.

The NanoGlue system has an onboard beam profiler and photodiode, both of which were used during alignment. The detector could be used by monitoring a DC signal or an AC signal in cases where interference fringes were being optimized. When using a DC signal, a feedback control system could be employed to perform "active" alignment. During assembly, a circulator was used on the input side of the module. This proved to be extremely useful in eliminating alignment variables of other components by maximizing power reflected back into the collimator. During alignment, a mirror (the same as the reference mirror) was used on the sample side. When optimizing alignment using interference patterns (or interferograms), an oscilloscope was used to maximize the interference amplitude. Specially designed alignment tools for fine-tuning the direction and position of the beams were manufactured. These were made from precision-machined aluminum and consisted of a $450-\mu \mathrm{m}$ diameter hole with a datum surface that was in contact with the corresponding datum surface on the substrate. This served to locate the position of the beam center consistently (within the machined tolerance of $\pm 10 \mu \mathrm{m}$ ) when the tool was in contact with the substrate. The alignment of optical components was based on achieving maximum power transmitted through the tool. Two alignment tools were utilized when necessary.

The choice of optical components was aided by the use of Zemax to model the beam characteristics into and out of each component. The working distances of the lenses were modeled, and the position of each of the components could be approximated. These measurements were imported into SolidWorks 3-D CAD software, where the prototype was developed for assembly.

The steps of alignment were as follows. The first component placed on the bench was the collimator. This was first glued to a mount, and the mount was then aligned with respect to the substrate. Two alignment tools were used to ensure that the collimated beam was both horizontal and not deviating from the optical axis. The on-board beam profiler was used to provide a feedback signal to optimize alignment. Next, the voice coil and reference mirror were aligned. The voice coil was fixed into its mount, and the reference mirror was glued to the barrel of the voice coil. The voice coil was actuated during alignment, and the input collimated beam was reflected straight back into the collimator by the reference mirror. The angle of the voice coil mount was optimized by maximizing the reflected signal observed at port 3 on the circulator signal. Next, the beam splitter was aligned. An alignment tool was placed on the turned path (sample arm), and by maximizing the signal observed by the beam profiler, the position and angle of the beam splitter were optimized. Next, a sample mirror was positioned offboard. This was mounted on a tip/tilt stage and a translation stage. It was placed at the zero-path length position, thereby allowing interference to be observed at the circulator signal using an oscilloscope. At this stage, the photodiode detector was aligned at the output side of the interferometer. Its output was connected to the transimpendance amplifier, and the interference pattern could then be monitored using this detector and an oscilloscope. The detector was held in place with one of the arms of the NanoGlue. A removable aluminum mount was screwed onto the sample side of the interferometer, and an optical blank was glued to it. This blank acts as dispersion compensation for the partial mirror. Next, the partial mirror was put in place. This was first roughly aligned using the circulator feedback and was then fine-tuned by maximizing the amplitude of the interference fringes and, most importantly, the number of orders seen. Typically, 20 orders could easily be seen. Moving the sample mirror through the orders of reflection and measuring the displacement tuned the position of the partial mirror relative to the position of the reference mirror. Next, the sample and reference lenses were aligned. Maximizing the DC signal seen on the circulator optimized the position of both of these. The detector lens was aligned to achieve maximum signal intensity on the photodiode detector. The lens, and then the detector, were glued into place.

\subsection{System Control and Experimental Set Up}

System control for the photonic module was done via a custombuilt FPGA board. The FPGA board interfaces with a PC to provide a user interface and display the acquired images. As with all time-domain OCT systems, the depth-information is achieved by mechanical movement of the reference mirror, which is mounted on the voice coil. Lateral scanning is achieved by mounting the module on a translation stage. The oscillation of the voice coil and synchronization with the lateral translation stages is controlled via the FPGA board. During data acquisition, the photonic module is mounted in the vertical orientation, so the voice coil is symmetrically loaded. The analog data are acquired and conditioned by the transimpedance amplifier. The data are then digitized by an analogue to digital converter. The FPGA is used to stream the data from the A to D to a laptop, via a USB 3 interface, where the data are processed. This is shown diagrammatically in Fig. 2.

\section{Multiple Reference Optical Coherence Tomography Processing}

The details of MR-OCT signal processing can be found in Dsouza et al.; $;^{7}$ however, the following is a brief explanation. Figure 3 shows a graphical representation of the processing steps used to visualize a single reflection from a plane mirror. For each sweep of the reference mirror, the photodetector captures the interference fringe pattern for all orders of reflection. Each order of reflection is distinct, in that both the effective scan range and the frequency of the respective interferogram are in direct proportion to the order number $n$. This increase in frequency with order is very convenient and is utilized in the processing steps to separate out each of the individual orders. Furthermore, the overlap of orders is defined by the scan range of the reference mirror $s$ and the distance between the reference mirror and partial mirror $d$ [both labeled in Fig. 1(a)].

First, the DC is removed from the raw interference signal. Figure 3(a) shows the interference signal for a single reflection from a sample mirror after DC removal. Note that there are two 


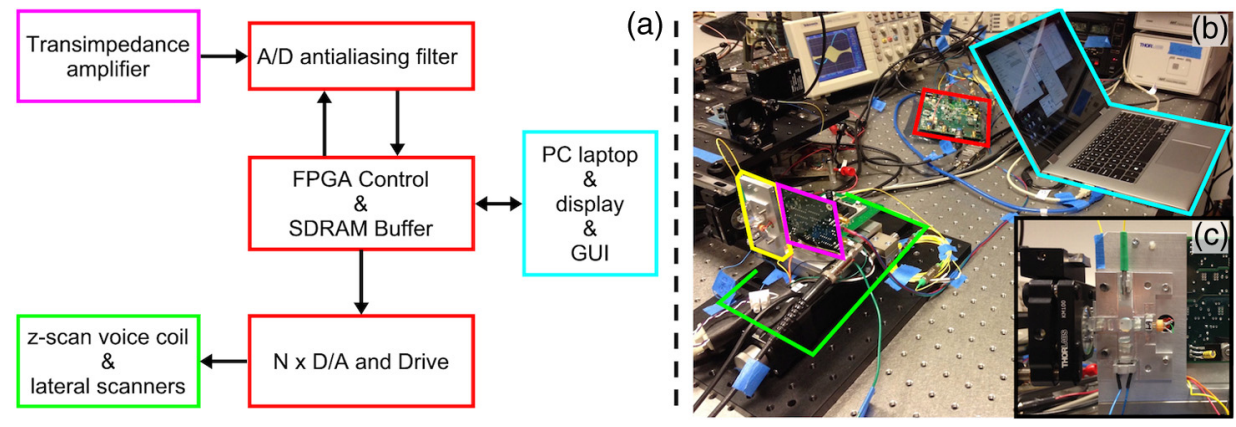

Fig. 2 (a) Schematic of the system control design. (b) Picture of the overview of the experimental set up. The critical system components are highlighted and correspond to the colors in (a). Note that the photonic module is highlighted in yellow. The inset image labeled (c) shows a close-up of the photonic module.
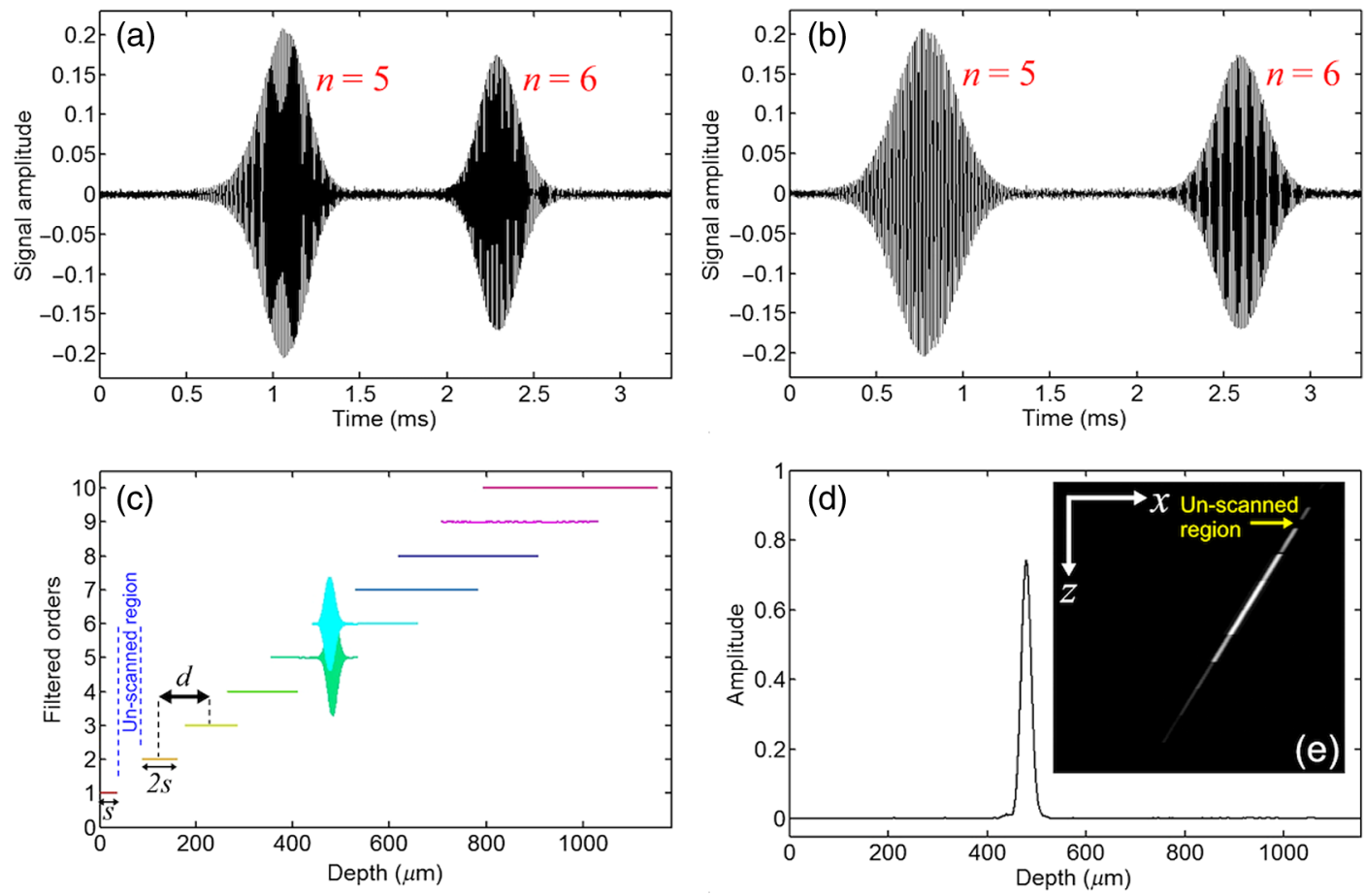

Fig. 3 (a) Raw interferograms of a single reflection from a sample mirror after removal of the DC signal. Two interferograms are present due to the mirror being at a position corresponding to the overlap of orders 5 and 6. (b) The interferograms after linearization. (c) Each order is separated by applying filtering thresholds due to the fact that each order has a distinct frequency. (d) Reconstructed A-line after demodulation and stitching of each of the individual orders. (e) Reconstructed movement of a sample reflector. The $x$-axis corresponds to position of the sample reflector and the $z$-axis corresponds to axial position or depth.

interferograms present because these involve reflections from orders 5 and 6 of the reference signal, and at this particular position, there is an overlap in space. The motion of the reference mirror is sinusoidal, so it is necessary to linearize the interferogram, the result of which is shown in Fig. 3(b). The time axes for Figs. 3(a) and 3(b) range one forward sweep of the reference mirror, i.e., half a period of oscillation. Note that these interferograms have different frequencies. Next, a polyphase filter bank is applied to separate out each of the individual orders, which can be seen in Fig. 3(c). The next step is signal demodulation, which is done by finding the envelope of the interferogram. The envelope corresponds to the amplitude of the reflected light from the sample and is found via the Hilbert transform. Finally, the demodulated signals from each order are stitched and reconstructed into a depth-dependent A-line, which is shown in Fig. 3(d).

A simple experiment, which is commonly performed for the calibration of MR-OCT systems, is done by acquiring A-lines of a single-reflecting surface (such as a mirror or optical blank) and stepping it toward or away from the system with successive scans. $^{7}$ If all of the processing steps outlined above are employed correctly, the resultant image is a clean, straight line. An example of the calibration of the miniature MR-OCT module used in this work can be seen in Fig. 3(e). In this experiment, 1024 A-lines were acquired and the sample reflector was stepped in distances of $2 \mu \mathrm{m}$. The scan range of the reference mirror is $30 \mu \mathrm{m}$, and the distance between the reference mirror and partial mirror is $98 \mu \mathrm{m}$. 

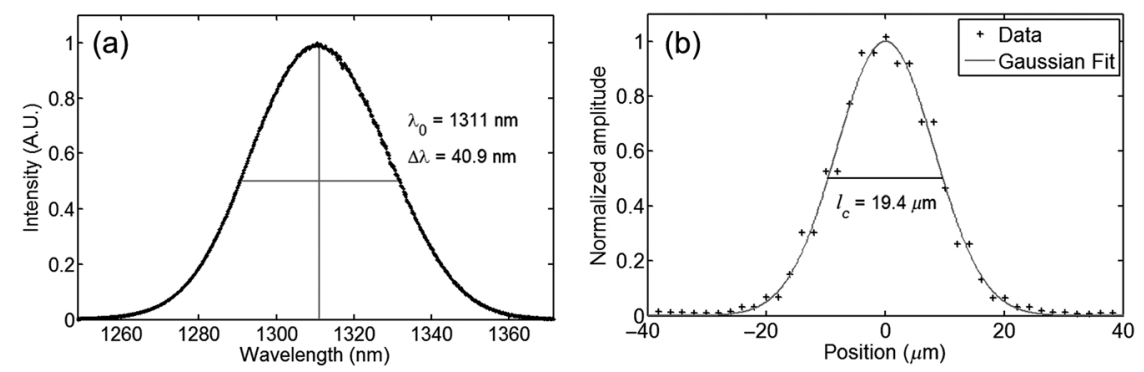

Fig. 4 (a) Input spectrum of the SLD. The central wavelength $\lambda_{0}=1311 \mathrm{~nm}$ and the full-width half-max bandwidth $\Delta \lambda=40.9 \mathrm{~nm}$. This results in a "round-trip" coherence length and axial resolution $I_{c}$ of $18.5 \mu \mathrm{m}$. (b) The coherence length was verified experimentally by measuring the full-width half-maximum of the point-spread function of a single A-line taken from a mirror.

\section{Results and Discussion}

\subsection{System Characterization}

The spectrum of the SLD used with this device was measured using a spectrometer and is shown in Fig. 4(a). A Gaussian function was fit to this profile, and a central wavelength, $\lambda_{0}$ of $1311 \mathrm{~nm}$ and a bandwidth, $\Delta \lambda$ of $40.9 \mathrm{~nm}$ were measured. Using these values, the "round trip" coherence length, $l_{\mathrm{c}} \sim 18.5 \mu \mathrm{m}$ (which corresponds to the axial resolution of the system) was approximated. This was verified experimentally by analyzing the axial point-spread function of a single reflector by using a plane mirror as the sample [Fig. 4(b)]. The definition of the round-trip coherence length is the full-width half-max of the point-spread function, ${ }^{23}$ which was measured to be $\sim 19.4 \mu \mathrm{m}$. The lateral resolution of the system is equal to the spot size of the scanning beam, which is $\sim 30 \mu \mathrm{m}$.

To characterize the signal-to-noise ratio (SNR) for each order, a mirror was used as the sample and was moved in depth through the scanning range. A-lines were acquired for each position of the sample, all of which are shown in Fig. 5(a).

For each A-line, a Gaussian function was fit to the data points and the maximum amplitude $A_{\max }$ was recorded in each case. An example of a single A-line with its Gaussian fit are shown in Fig. 5(b). A "noise" A-line was also acquired when no sample was present and the standard deviation $\sigma$ was calculated. The SNR for each mirror position was calculated in decibel according to the following equation:

$\mathrm{SNR}=20 \log _{10}\left(\frac{A_{\text {max }}}{\sigma_{\text {noise }}}\right)$, and are shown in Fig. 5(c). In the case of overlapping regions, the lower order A-line was used. Dsouza et al. have previously shown that the sensitivity of a benchtop MR-OCT falls off linearly (in $\mathrm{dB}$ ) at a rate of $1.1 \mathrm{~dB}$ per order. ${ }^{7}$ The primary explanation for this is that the reference intensity for each order decreases as a function of the transmission percentage of the partial mirror, i.e., a higher transmission value of the partial mirror will cause the intensity to fall off at a higher rate per order. However, the result shown in Fig. 5(c) implies a maximum SNR at a depth of $\sim 400 \mu \mathrm{m}$. A possible explanation for this is that the focal plane of the sample lens occurs at this depth and the fall-off of SNR at shallower and deeper depths is due to the confocal parameter of the lens. In all applications, it is beneficial to align the system such that the focal plane occurs at the depth of interest to maximize the use of the confocal parameter.

\subsection{Sample Images}

Examples of the imaging capabilities of the miniature MR-OCT device can be seen in Fig. 6. Figure 6(a) shows an image of the cross section of a roll of highly scattering translucent tape. The individual layers of the tape can clearly be seen. Figure 6(b) shows the cross section of the surface of a metallic screw. This is relevant to applications of MR-OCT such as nondestructive testing where cost-effective means of detecting scratches and defects on objects produced in high-volume are necessary. Figures 6(c) and 6(d) show in vivo images of the human finger. [All experiments were approved by the ethics committee of the Galway University Hospitals (Ref: C.A. 1053).] Figure 6(c) shows the palmer region of the finger-tip. The epidermis and
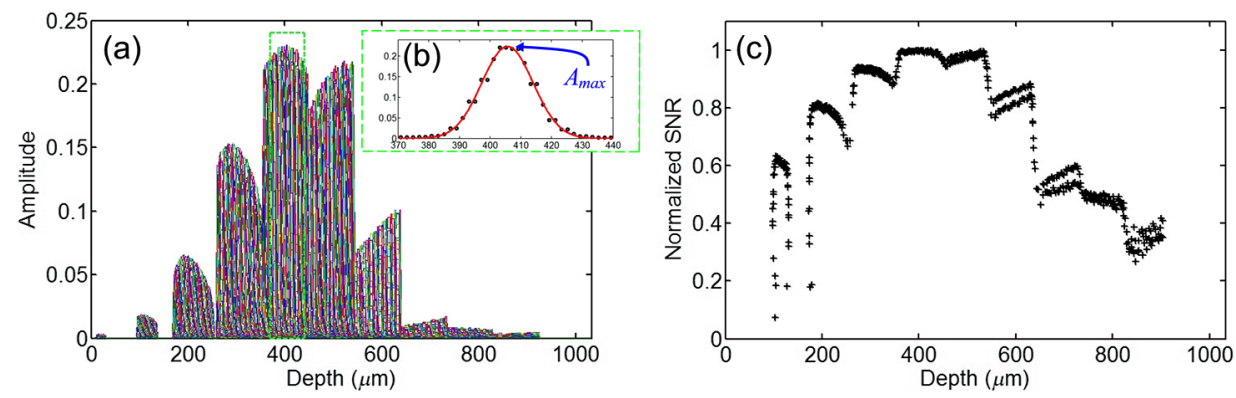

Fig. 5 (a) A-lines of a mirror were acquired at multiple depths. (b) An example of a single A-line with a Gaussian fit. The maximum amplitude is used to calculate the SNR. (c) Normalized SNR for each position of the sample mirror. 

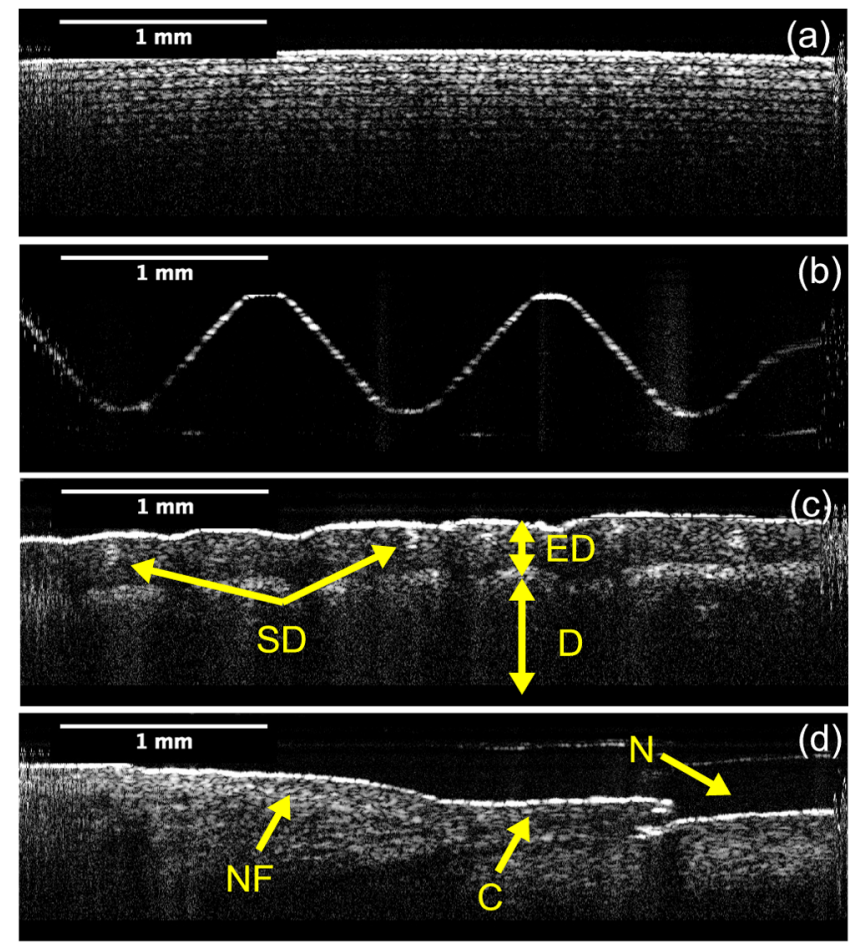

Fig. 6 B-frames of various samples. These images have dimensions of $4 \mathrm{~mm}$ across by $\sim 1.1 \mathrm{~mm}$ in depth. (a) Highly scattering translucent tape. (b) Surface of a metal screw. (c) Human in-vivo finger-tip. Note that interesting structural features such as sweat ducts (SD) and the dermal (D) and epidermal (ED) layers can easily be identified. (d) Human in-vivo nail fold (NF) region of the finger. The NF, cuticle (C), and nail $(\mathrm{N})$ can easily be distinguished.

dermis layers of the tissue can clearly be distinguished (labeled "ED" and "D"). The undulations of the surface of the skin are seen replicated in the deeper dermis-epidermis boundary. This is the subsurface primary fingerprint to which reference was made in the introduction of this paper. Furthermore, the presence of eccrine sweat ducts, which transport sweat to the surface of the skin, can be seen. These appear as bright spiral features and are labeled "SD." Figure 6(d) shows the nailfold region of the finger. The nail $(\mathrm{N})$, cuticle $(\mathrm{C})$, and nail fold $(\mathrm{NF})$ regions can clearly be distinguished.

\section{Conclusions}

This work has demonstrated the assembly and alignment of a miniature MR-OCT imaging system with a dynamic range and sensitivity appropriate for imaging highly scattering media including human tissue. The in vivo images shown in the results section are especially relevant for applications of this technology to biometric security via subsurface finger print imaging. As a first iteration, significant progress has been made in terms of miniaturization. It is expected that this can be progressed further to a target footprint similar to that of the pick-up head contained within a CD/DVD player. All of the components used in this device are available off-the-shelf, meaning that the potential bill-of-materials for an assembly of a miniature module such as this can be kept very low, especially in large quantities.

It is important to highlight that the voice coil motor used in this device is not design-specific. This component is designed for positioning a focusing lens in front of a camera sensor on a smartphone and is not designed to be driven continuously at high frequency. Despite this, this component has performed way beyond expectations and has fulfilled the purpose of demonstrating that off-the-shelf components are feasible for use in an MR-OCT device. Currently, a design-specific voice coil with superior pointing stability, which operates at higher frequencies, is under development. Furthermore, it is worth nothing that for this work, only the forward sweep of the reference mirror was used. It is entirely feasible to utilize both forward and backward sweeps to effectively double the speed of acquisition.

The alignment of a miniature MR-OCT device is extremely important and can impose limitations on the detection sensitivity of a particular device. An advantage of the polarized version of the device is the ability to perform balanced detection; however, the additional optical components (polarizers, wave-plates, detector) means assembly and alignment becomes more complex. Inevitably, the optimal configuration of MR-OCT depends on the application (e.g., a polarized design is not necessary for ophthalmic applications due to minimal scattering).

The next phase of this work will attempt to mount the light source directly on the module. There are numerous ways in which this can be accomplished, and both TO can mounted and chip-on-submount SLD designs will be investigated. The contribution of the cost of an on-board SLD to the overall bill-of-materials is an important point of discussion. Currently, SLDs can cost in the range of $\$ 1500$, but there has never been a market for high volume production. Our argument is that finding an appropriate high-volume application will provide the path to reducing the cost of the SLD to an amount comparable to that of the laser diode.

Finally, the issue of lateral scanning is being looked at in numerous ways. It could be possible to implement internal scanning through the introduction of scanning mirrors in the path of the sample beam, as is common in traditional OCT architectures. A simpler approach is, by further reducing the dimensions of the device to those comparable to a pick-up head contained within a CD/DVD player, it would be feasible to laterally scan the whole device (as demonstrated in this work). Alternatively, parallel detection, which has previously been shown to be effective with other modalities of OCT, ${ }^{24,25}$ is also a possibility.

\section{Disclosures}

The TOMI group at NUI Galway has received research support from Compact Imaging, Inc. P. McNamara, R. Dsouza, C. Wilson, J. Hogan, and M. Leahy have a financial interest in Compact Imaging, Inc. and the intellectual property of MR-OCT. C. O'Riordan, S. Collins, and P. O'Brien declare no relevant financial interests.

\section{Acknowledgments}

This work was supported by the Irish Research Council and Compact Imaging Inc. under the Enterprise Partnership Scheme, 2014. Additional funding was provided by the Science Foundation Ireland/IPIC (Irish Photonic Integration Centre), Galway University Foundation, the University of Limerick Foundation, the National Biophotonics Imaging Platform (NBIP) Ireland funded under the Higher Education Authority PRTLI Cycle 4, cofunded by the Irish Government, and the European Union-investing in your future. 


\section{References}

1. D. Huang et al., "Optical coherence tomography," Science 254(5035), 1178-1181 (1991).

2. A. M. Zysk et al., "Optical coherence tomography: a review of clinical development from bench to bedside," J. Biomed. Opt. 12(5), 051403 (2007).

3. B. I. Akca et al., "Miniature spectrometer and beam splitter for an optical coherence tomography on a silicon chip," Opt. Express 21(14), 16648-16656 (2013).

4. G. Yurtsever et al., "Photonic integrated Mach-Zehnder interferometer with an on-chip reference arm for optical coherence tomography," Biomed. Opt. Express 5(4), 1050-1061 (2014).

5. Z. Wang et al., "Silicon photonic integrated circuit swept-source optical coherence tomography receiver with dual polarization, dual balanced, in-phase and quadrature detection," Biomed. Opt. Express 6(7), 2562-2574 (2015).

6. G. Yurtsever et al., "Ultra-compact silicon photonic integrated interferometer for swept-source optical coherence tomography," Opt. Lett. 39(17), 5228-5231 (2014).

7. R. Dsouza et al., "Dermascope guided multiple reference optical coherence tomography," Biomed. Opt. Express 5(9), 2870-2882 (2014).

8. R. Dsouza et al., "Assessment of curing behavior of light-activated dental composites using intensity correlation based multiple reference optical coherence tomography," Lasers Surg. Med. 48(1), 77-82 (2015).

9. R. Dsouza et al., "3D nondestructive testing system with an affordable multiple reference optical-delay-based optical coherence tomography," Appl. Opt. 54(18), 5634-5638 (2015).

10. M. J. Leahy et al., "The how and why of a $\$ 10$ optical coherence tomography system," Proc. SPIE 9697, 96970T (2016).

11. H. S. G. da Costa et al., "Evaluation of fingerprint deformation using optical coherence tomography," Proc. SPIE 8946, 89460I (2014).

12. A. Bossen, R. Lehmann, and C. Meier, "Internal fingerprint identification with optical coherence tomography," IEEE Photonics Tech. Lett. 21(7), 507-509 (2010).

13. L. N. Darlow, J. Connan, and S. S. Akhoury, "Internal fingerprint zone detection in optical coherence tomography fingertip scans," J. Electron. Imaging 24(2), 023027 (2015).

14. A. Zam et al., "Feasibility of correlation mapping optical coherence tomography (cmOCT) for anti-spoof sub-surface fingerprinting," J. Biophotonics 6(9), 663-667 (2013).

15. M. Y. Liu and T. Buma, "Biometric mapping of fingertip eccrine glands with optical coherence tomography," IEEE Photonic Tech. Lett. 21(22), 1677-1679 (2010).

16. G. Liu and Z. Chen, "Capturing the vital vascular fingerprint with optical coherence tomography," Appl. Opt. 52(22), 5473-5477 (2013).

17. C. A. Puliafito et al., "Imaging of macular diseases with optical coherence tomography," Ophthalmology 102(2), 217-229 (1995).

18. M. R. Hee et al., "Optical coherence tomography of age-related macular degeneration and choroidal neovascularization," Ophthalmology 103(8), 1260-1270 (1996).

19. P. A. Keane et al., "Evaluation of age-related macular degeneration with optical coherence tomography," Surv. Ophthalmol. 57(5), 389-414 (2012).

20. J. Hogan, "Multiple reference OCT system," U.S. Patent 9,113,782 (2015).

21. S. Meissner, R. Breithaupt, and E. Koch, "Defense of fake fingerprint attacks using a swept source laser optical coherence tomography setup," Proc. SPIE 8611, 86110L (2013).

22. M. Leahy et al., "Multiple reference optical coherence tomography (MR-OCT) system," Proc. SPIE 8580, 85800L (2013).

23. J. A. Izatt and M. A. Choma, "Theory of optical coherence tomography," in Optical Coherence Tomography: Technology and Applications, W. Drexler and J. G. Fujimoto, Eds., pp. 47-72, Springer, Berlin, Heidelberg (2008).

24. D. J. Fechtig et al., "Line-field parallel swept source MHz OCT for structural and functional retinal imaging," Biomed. Opt. Express 6(3), 716-735 (2015).
25. R. N. Graf, W. J. Brown, and A. Wax, "Parallel frequency-domain optical coherence tomography scatter-mode imaging of the hamster cheek pouch using a thermal light source," Opt. Lett. 33(12), 1285-1287 (2008).

Paul M. McNamara received his PhD in biophotonics in 2013 from the University of Limerick, specializing in tissue viability (TiVi) imaging and full-field optical coherence tomography. $\mathrm{He}$ is a postdoctoral researcher at the Tissue Optics and Microcirculation Imaging Group at NUI Galway and also Head of Application Research Ireland for Compact Imaging Ireland, Ltd. His research interests include wearable devices and biophotonics applications in low-resource settings.

Roshan Dsouza received his master's degree in photonics from Manipal University, India, in 2011 and his $\mathrm{PhD}$ in biomedical optics from the National University of Ireland, Galway, in 2016. From 2015 to 2016, he worked as a research associate at Compact Imaging Ireland, Ltd. He currently is a postdoctoral research associate at the Beckman Institute for Advance Science and Technology, University of Illinois at Urbana-Champaign. His research interests include biomedical optics and development of low-cost imaging systems.

Colm O'Riordan received his bachelor's degree in electronic engineering and his $\mathrm{PhD}$ in photonics from the University of Limerick, Ireland. He was a project manager and design lead at IPIC on the miniature MR-OCT project. Previous experience includes positions as a postdoctoral researcher at Tyndall National Institute investigating flexible optical frequency comb sources and a senior optical design engineer at Nualight developing LED-based illumination systems. $\mathrm{He}$ is currently an optoelectronic engineer with Tomra Sorting, Dublin.

Seán Collins received his bachelor's degree in biomedical engineering from the University of Limerick, Ireland, in 2009. He is currently an engineer in the Photonics Packaging Group, Tyndall National Institute, Ireland, specializing in the mechanical design and assembly of photonic packages.

Peter O'Brien received his $\mathrm{PhD}$ in physics from the University College Cork. He was a postdoctoral scholar at the California Institute of Technology and research scientist at NASA's Jet Propulsion Laboratory. He founded two photonic companies and established the Photonics Packaging Group at Tyndall in 2009. He performs research in the area of integrated photonics, in particular, the development of scalable packaging processes for volume manufacturing in applications including medical devices, fiber optic communications, and sensors.

Carol Wilson holds a doctorate in mechanical engineering from UC Berkeley. She is a cofounder and VP of engineering at Compact Imaging, Inc. Previously, she developed low-cost small optoelectronics systems at HP Labs, Agilent, and IBM (IBM Fellow). She is named inventor on multiple patents and has won awards for her technical achievements.

Josh Hogan holds a doctorate in solid state physics from Ulster University. He is a founder and CTO of Compact Imaging, Inc. $\mathrm{He}$ holds over 50 patents, including several on compact imaging's MR-OCT technology. Previously, he developed low-cost, small optoelectronic technologies, including DVD_RW optical storage systems, at HP Labs, Palo Alto, California.

Martin J. Leahy graduated with a DPhil in laser-based instrumentation for the measurement of blood flow and oxygenation from the University of Oxford in 1997. He is the head of the Tissue Optics and Microcirculation Imaging (TOMI) Group at NUI Galway. He is an adjunct professor at the Royal College of Surgeons, fellow of the Institute of Physics, Ireland, fellow of the Royal Academy of Medicine, Ireland, and fellow of SPIE. 